\title{
Local Infiltration of Bupivacaine versus Placebo for Post-Operative Pain Relief Following Elective Lichtenstein Hernioplasty: A Randomized Controlled Trial
}

\author{
Arshad Rashid $^{1^{*}}$ (D), Mubasher Bhat ${ }^{2}$ (D) Sheikh Junaid ${ }^{3}$ (D), Majid Mushtaque ${ }^{3}$ (D) \\ ${ }^{1}$ Lecturer, Department of Surgery, Government Medical College \& Associated Hospitals, Srinagar - 190010, India \\ ${ }^{2}$ Assistant Professor, Department of Anaesthesiology \& Pain Management, Government Medical College \& Associated Hospitals, Srinagar \\ - 190010, India \\ ${ }^{3}$ Surgeon Specialist, Health Services Kashmir - 190001, India \\ * Corresponding author: Arshad Rashid E-mail: arsh002@gmail.com ORCID: 0000-0002-7109-7773 \\ Received: 30 April 2020 Accepted: 27 May 2020
}

\begin{abstract}
Introduction: Postoperative pain relief is of paramount importance in any surgery.

Objective: To compare the effect of local infiltration of bupivacaine versus placebo for post-operative pain relief following inguinal hernioplasty.

Materials and Methods: Ours was a prospective randomized controlled trial conducted on 120 patients undergoing elective Lichtenstein hernioplasty. Patients were divided into two groups of 60 each. One group received local infiltration with $10 \mathrm{~mL}$ bupivacaine $0.25 \%$ below the external oblique aponeurosis and $15 \mathrm{~mL}$ bupivacaine $0.25 \%$ subcutaneously after completion of the surgical procedure. The other group received an equal amount of normal saline infiltrated in the similar manner. Visual Analogue Scale measured the pain.
\end{abstract}

Results: The baseline parameters were similar in both the groups. Postoperative pain was significantly decreased in patients receiving bupivacaine $(p<0.05)$. Mean time to first request for rescue analgesic was increased from $245+19.42$ minutes to $472+19.42$ minutes $(p<0.05)$. The consumption of supplementary analgesics during the 24 -hour study period reduced from a mean of $2.8+0.12$ to $1.7+0.09$ doses in bupivacaine group $(p<0.05)$.

Conclusion: Local infiltration of bupivacaine is an effective modality for post-operative pain relief in inguinal hernioplasty.

Keywords: bupivacaine, analgesia, post-operative, hernia, lichtenstein

\section{INTRODUCTION}

Pain is defined by International Association for the Study of Pain as "an unpleasant sensory and emotional experience associated with actual or potential tissue damage" [1]. Any surgical procedure causes postoperative pain that should be alleviated as soon and as effective as possible to reduce

( 2020 by the authors; licensee EJMETS by Bastas, UK. This article is an open access article distributed under the terms and conditions of the Creative Commons Attribution License (http://creativecommons.org/licenses/by/4.0/). 


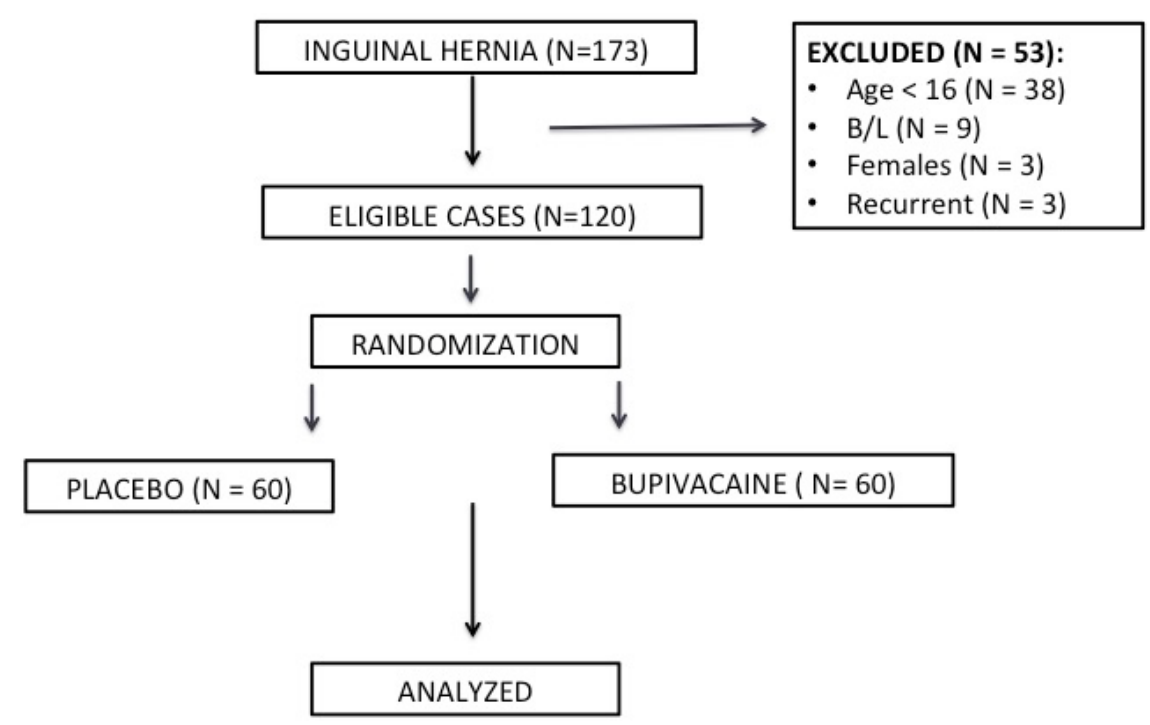

Figure 1. The CONSORT flow diagram of the study

suffering, to promote the healing process to prevent complications. However, clinical pain management after surgery is far from being successful. It is a well-documented fact that post-operative pain is undertreated [2]. Opioids are the mostly used medicine for post-surgical pain control [3]. Among the many other options available for the control of post-operative pain, analgesia delivered through an indwelling epidural catheter or local infiltration of wound site by an anesthetic agent is a safe and effective method for the management of acute postoperative pain [2].

Inguinal hernia repair is one of the most commonly performed surgical procedures. In fact, it is the bread and butter of surgeons. Approximately 800,000 are performed each year in the United States, 100,000 in France, and 80,000 in the United Kingdom [4]. Most patients suffer from pain after inguinal hernia surgery and many develop chronic pain after surgery, which might be, at least in part, a result of undertreated acute postoperative pain [5]. Bupivacaine is a potent agent that belongs to the amide group of local anaesthetics and blocks the generation of pain related action potentials by increasing the threshold of the nerve fibers [6]. The aim of the present study was to compare local infiltration of bupivacaine versus normal saline for postoperative pain relief after inguinal hernioplasty.

\section{MATERIALS AND METHODS}

Ours was a prospective randomized controlled trial comprising of a cohort of patients undergoing Lichtenstein hernioplasty with the primary outcome of comparing the post-operative analgesic effects of bupivacaine infiltration versus placebo. Between March 2017 and March 2020, a total of 173 patients with groin hernias were treated by Lichtenstein tension free repair at a district level hospital of
Health Services Kashmir. The sample size of the study was not calculated. All of the cases were admitted on an elective basis and only the uncomplicated hernias were taken up for the study. Exclusion criteria included patients with bilateral hernias, recurrent hernias, females, age less than 16 years and those with who refused to participate in the study. All of the patients were advised to empty their urinary bladder just before surgery. The patients were operated under spinal anaesthesia.

After excluding the patients who were not meeting the study criteria, we were left with 120 eligible patients. The patients were randomly divided into two groups of 60 each by a computer-generated table of random numbers (Figure 1). The first author did all the surgical procedures. The hernia defects were classified in accordance with the EHS classification and the defect sizes were measured intraoperatively. After hernioplasty, the first group received infiltration with $10 \mathrm{~mL}$ bupivacaine $0.25 \%$ below the external oblique aponeurosis (under ultrasound guidance) and $15 \mathrm{~mL}$ bupivacaine $0.25 \%$ subcutaneously after completion of the surgical procedure. The other group received an equal amount of normal saline infiltrated in the similar manner. Identical containers were used for bupivacaine and saline as a method of allocation concealment. Postoperative pain was measured by visual analog scale (VAS). The pain scores were obtained at 1, 2, 4, $6,8,12$ and 24 hours postoperatively. Rescue analgesics (Diclofenac $50 \mathrm{mg}$ tablet) were administered if the VAS score was $>6$ or on demand. The total diclofenac consumption and time to first request for rescue analgesic were recorded. As the primary endpoint of this study was immediate postoperative pain and analgesic requirement, so long-term follow-up was not done. 
Table 1. Baseline Parameters of the two groups

\begin{tabular}{|c|c|c|c|c|}
\hline \multicolumn{2}{|c|}{ PARAMETER } & BUPIVACAINE $(n=60)$ & PLACEBO $(n=60)$ & P Value \\
\hline \multicolumn{2}{|c|}{ Mean Age (Years) } & $53.34 \pm 9.42$ & $55.72 \pm 8.39$ & 0.9821 \\
\hline \multicolumn{2}{|c|}{ Mean BMI $\left(\mathrm{Kg} / \mathrm{m}^{2}\right)$} & $22.45 \pm 0.22$ & $23.02 \pm 0.31$ & 0.8665 \\
\hline \multirow{3}{*}{$\begin{array}{l}\text { European Hernia Society (EHS) Type } \\
\text { (N) }\end{array}$} & Medial & $11(18.33 \%)$ & $13(21.67 \%)$ & 0.9132 \\
\hline & Lateral & $40(66.67 \%)$ & $39(65 \%)$ & 0.8954 \\
\hline & Combined & $9(15 \%)$ & $8(13.34 \%)$ & 0.8748 \\
\hline \multirow{3}{*}{ Defect Size (N) } & $\mathrm{I}(<1.5 \mathrm{~cm})$ & $10(16.67 \%)$ & $11(18.34 \%)$ & 0.9231 \\
\hline & II $(1.5-3 \mathrm{~cm})$ & $42(70 \%)$ & $43(71.67 \%)$ & 0.8986 \\
\hline & III $(>3 \mathrm{~cm})$ & $8(13.34 \%)$ & $6(10 \%)$ & 0.5324 \\
\hline
\end{tabular}

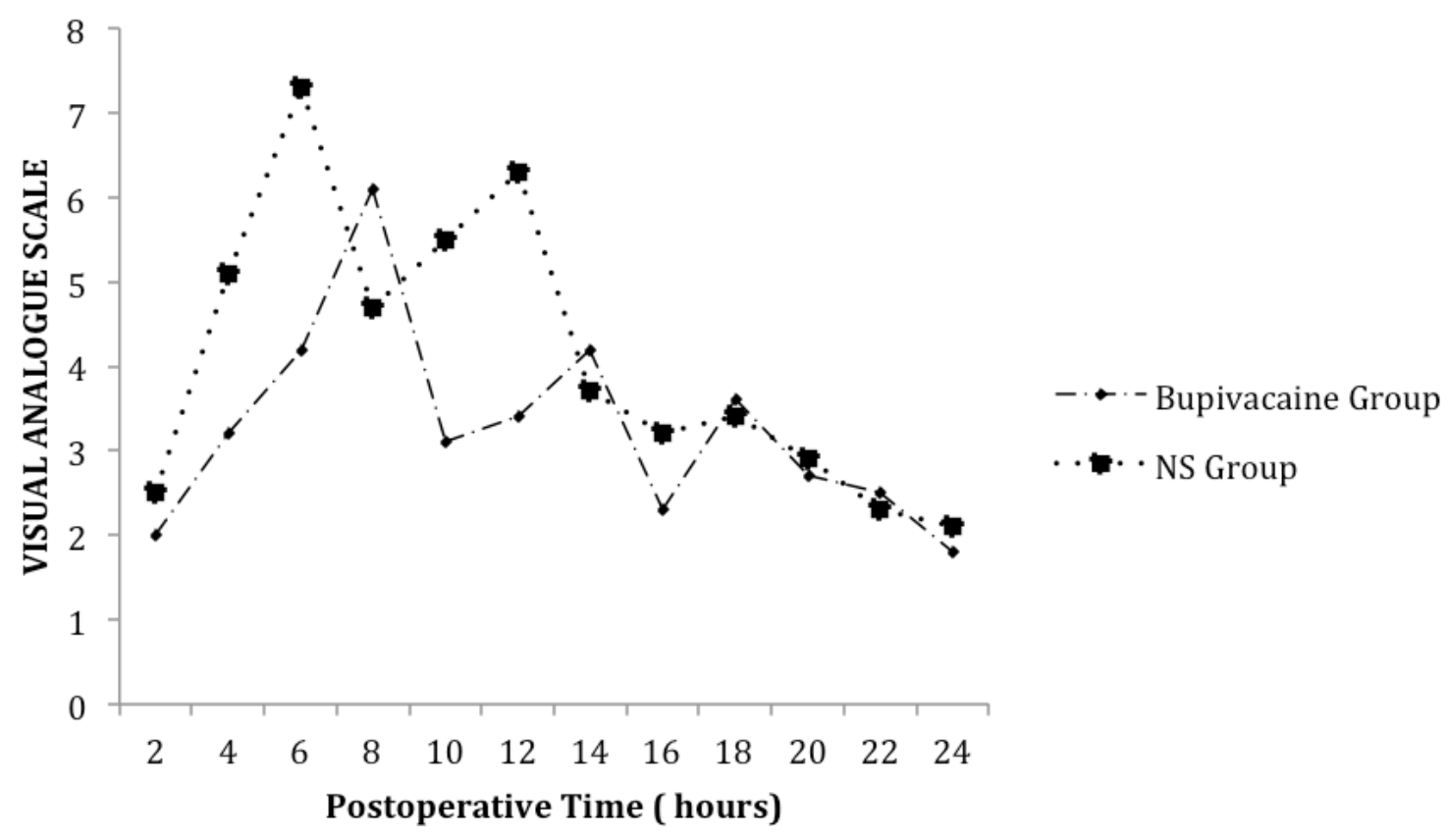

Figure 2. The pain scores (VAS) in the two groups over 24 hours revealed a significant difference $(P<0.05)$

An approval from the institutional ethical committee was obtained for the purpose of this study. This clinical trial was registered with the local trials registry (DHB-13 of 2017). A written and informed consent was taken from the patients for publication and analysis of their data after explaining to them the protocol of the study in their own language. The data thus collected was compiled and analyzed using SPSS version 22 for Mac (IBM Corporation, 2012). To calculate the P-value, Fisher's exact test and Pearson's chi-square test were applied to compare the frequencies for categorical parameters, and the unpaired t-test was used to compare the means (2-tailed) among continuous variables. A P-value $<0.05$ was considered significant.

\section{RESULTS}

The baseline parameters were similar in both the groups and all efforts were made to minimize the confounding factors (Table 1). Our study included cases of direct, indirect and combined uncomplicated primary inguinal hernias. All the cases were operated under spinal anesthesia. Postoperative pain was significantly decreased in patients receiving bupivacaine compared to saline (Figure 2, p < 0.05). There was no difference in the post-operative hospital stay of the two groups ( $p>0.05)$, and almost all the patients in either of the groups were discharged on the first post-operative day.

There were two peaks above VAS score of 6 in the placebo group as compared to only one peak in the bupivacaine group. Mean time to first request for rescue analgesic was increased from $245 \pm 19.42$ minutes to $472 \pm 19.42$ minutes $(p<0.05)$ in the bupivacaine group. The consumption of supplementary analgesics during the 24-hour study period reduced from a mean of $2.8 \pm 0.12$ to $1.7 \pm 0.09$ doses in bupivacaine group as compared to saline group ( $<0.05)$. No untoward side effects were noted in either of the groups and none of our patients developed a seroma.

\section{DISCUSSION}

To the serious and dedicated surgeon, it would be unthinkable to expect a career without being competent enough in the performance of repair for inguinal hernias. Accounting for $75 \%$ of all abdominal wall hernias, and with a lifetime risk of $27 \%$ in men and $3 \%$ in women, inguinal hernia repair is one of the most commonly performed surgery in the world $[7,8]$. It is imperative that such a surgery 
remains pain free in the post-operative period. Optimizing postoperative analgesia improves clinical outcomes and increases patient satisfaction while uncontrolled postoperative pain can result in significant morbidity and mortality. Infiltration of wounds with local anesthetics not only provides analgesia but also appears to reduce the local inflammatory response to trauma or surgery [3]. The current paper is an attempt to determine the effect of bupivacaine infiltration on post-operative pain in patients with elective inguinal hernioplasty.

The aim of post-operative analgesia is to provide subjective comfort with minimum side effects [9]. It also helps to blunt autonomic and somatic reflex responses to pain and allows early ambulation and restoration of function. If the postoperative pain is not controlled, it leads to detrimental acute and chronic effects [10]. The nociceptive input to the central nervous system leads to activation of neuroendocrine stress response mainly involving the hypothalamic-pituitaryadrenocortical axis. This results in tachycardia, hypertension, and increased myocardial oxygen consumption [11]. In addition, it can decrease the myocardial oxygen supply through coronary vasoconstriction leading to myocardial ischemia and infarction in vulnerable patients. The metabolic changes occurring lead to sodium and water retention, increased blood glucose, and lactate levels. Hyperglycemia leads to poor wound healing and immunosuppression. Nausea and vomiting occur due to nociceptive impulses from viscera and somatic structures [10-12].

The major causes of postoperative pain are the stretching of the wound during surgery and the total length of the fascial incision [13]. Incision leads to an acute inflammatory response in which neutrophils, mast cells and other cells are recruited that secretes various cytokines and lead to stimulation of nociceptors. The stretching of the wound also leads to activation and sensitization of peripheral nociceptors and spinal dorsal horn neurons [5]. Bupivacaine is a long acting local anesthetic agent, which helps in pain alleviation by blocking these nociceptive impulses. In a study by Dierking et al, pain at rest, during mobilization and during cough was significantly decreased in patients receiving bupivacaine compared to placebo [14]. They reported that median time to first request for morphine was increased from 25 minutes to 135 minutes, and the consumption of supplementary morphine during the twenty-four hour study period reduced from four to two doses of $0.1 \mathrm{mg} / \mathrm{kg}$ IV or $0.125 \mathrm{mg} / \mathrm{kg} \mathrm{IM}$, in patients who received bupivacaine compared to placebo [15]. Ding et al advocated the use of an ilioinguinal-hypogastric nerve block with bupivacaine $0.25 \%$ as an adjuvant during inguinal herniorrhaphy under monitored anaesthesia care for decreasing the pain and analgesic requirements [16]. Our study supplemented these results. Less postoperative pain demonstrated added advantages as it translated into early ambulation, acceptance of orals, and discharge of patients in the bupivacaine group.

In our study, the consumption of supplementary analgesics during the twenty-four hour study period reduced from a mean of $2.8 \pm 0.12$ to $1.7 \pm 0.09$ doses in bupivacaine group as compared to saline group. Harrison et al reported that the combination of nerve block and wound infiltration reduced consumption of morphine in the first twenty-four hours after herniorrhaphy, but they failed to show any effect of $0.5 \%$ bupivacaine beyond the first six hours after operation [17]. Our patients demonstrated a sustained effect as the first dose of rescue analgesia was given at an average time of $472 \pm 19.42$ minutes in the bupivacaine group as compared to $245 \pm 23.17$ minutes in the saline group. The maximum scores of pain were observed at 4 hours postoperatively in the placebo group, presumably due to exhaustion of the effects of spinal anesthesia; however, the maximum scores of pain were observed at 8 hours postoperatively in the bupivacaine group.

The present study has some potential limitations. The sample size was small and the population was homogenous, so extrapolation of results to other parts of the globe may not be valid. Our study was not a double-blinded one. However, the postoperative pain assessment was somewhat blinded as all scoring was performed by the attending nurse who was unaware of the ongoing study. Our study can also be criticized on the plea that cost/benefit ratio was not done. We did not do it as bupivacaine and diclofenac was available free of cost in the hospital as per the hospital health policy. The strength of our study is it being randomized and thus eliminating selection bias.

\section{CONCLUSION}

Local infiltration of bupivacaine is an effective modality for post-operative pain relief after Lichtenstein inguinal hernioplasty. It also helps to reduce the overall postoperative systemic pain medication.

\section{DECLARATION OF CONFLICT OF INTEREST}

The authors received no financial support for the research and/or authorship of this article. There is no conflict of interest. 


\section{REFERENCES}

1. Mehta S, Gajbhare MN, Kamble NP. Comparison of Epidural Analgesia Using 0.2\% Bupivacaine and 0.2\% Ropivacaine for the Management of Postoperative Pain in Major Orthopedic Surgery. Anesth Essays Res. 2018; 12(2): 586-91. (doi: 10.4103/aer.AER_62_18).

2. Gan TJ, Habib AS, Miller TE, White W, Apfelbaum JL. Incidence, Patient satisfaction, and perceptions of postsurgical pain: Results from a US national survey. Curr Med Res Opin. 2014; 30: 149-60. (doi: 10.1185/03007995.2013.860019).

3. Barawi SAM, Saleh SA. Local injection of bupivacaine hydrochloride to reduce postoperative pain in obstetrical and gynaecological surgical incision. J Anesth Clin Res. 2017; 8(10): 768. (doi: 10.4172/21556148.1000768).

4. HerniaSurge Group. International guidelines for groin hernia management. Hernia. 2018; 22(1): 1-165. (doi: 10.1007/s10029-017-1668-x).

5. Pogatzki-Zahn EM, Segelcke D, Schug SA. Postoperative pain-from mechanisms to treatment. Pain Rep. 2017; 2(2): e588. (doi: 10.1097/PR9.0000000000000588).

6. Shah J, Votta-Velis EG, Borgeat A. New local anesthetics. Best Pract Res Clin Anesthesiol. 2018; 32(2): 179-85. (doi: 10.1016/j.bpa.2018.06.010).

7. Miserez M, Peeters $E$, et al. Update with level 1 studies of the European Hernia Society guidelines on the treatment of inguinal hernia in adult patients. Hernia. 2014; 18: 151 63. (doi: 10.1007/s10029-014-1236-6).

8. Miserez M, Alexandre JH, Campanelli G, et al. The European hernia society groin hernia classification: simple and easy to remember. Hernia. 2007; 11(2): 113-6. (doi: 10.1007/s10029-007-0198-3).

9. Patil SS, Kudalkar AG, Tendolkar BA. Comparison of continuous epidural infusion of $0.125 \%$ ropivacaine with $1 \mu \mathrm{g} / \mathrm{ml}$ fentanyl versus $0.125 \%$ bupivacaine with 1 $\mu \mathrm{g} / \mathrm{ml}$ fentanyl for postoperative analgesia in major abdominal surgery. J Anaesthesiol Clin Pharmacol. 2018; 34(1): 29-34. (doi: 10.4103/joacp.JOACP_122_16).
10. Dumlu EG, Tokaç M, Öcal H, et al. Local bupivacaine for postoperative pain management in thyroidectomized patients: A prospective and controlled clinical study. Ulus Cerrahi Derg. 2015; 32(3): 173-7. (doi: 10.5152/UCD.2015.3138).

11. Brennan TJ. Pathophysiology of postoperative pain. Pain. 2011; 152(3 Suppl): S33-S40. (doi: 10.1016/j.pain.2010.11.005).

12. Brennan TJ, Zahn PK, Pogatzki-Zahn EM. Mechanisms of incisional pain. Anesthesiol Clin North America. 2005; 23: 1-20. (doi: 10.1016/j.atc.2004.11.009).

13. Rashid A, Nazir S, Kakroo MS, Chalkoo MA, Razvi SA, Wani AA. Laparoscopic Interval Appendectomy Versus Open Interval Appendectomy: A prospective randomized controlled trail. Surg Laparosc Endosc Percutan Tech. 2013;23(01): 93-6. (doi: 10.1097/SLE.0b013e318277df6a).

14. Dierking GW, Ostergaard E, Ostergard HT, Dahl JB. The effects of wound infiltration with bupivacaine versus saline on postoperative pain and opioid requirements after herniorrhaphy. Acta Anaesthesiol Scand. 1994; 38(3): 289-92. (doi: 10.1111/j.1399-6576.1994.tb03891.x).

15. Dierking GW, Dahl JB, Kanstrup J, Dahl A, Kehlet H. Effect of pre- versus postoperative inguinal field block on postoperative pain after herniorrhaphy. $\mathrm{Br} J$ Anaesth. 1992; 68(4): 344-8. (doi: 10.1093/bja/68.4.344).

16. Ding Y, White PF. Post-herniorrhaphy pain in outpatients after pre-incision ilioinguinal-hypogastric nerve block during monitored anaesthesia care. Can J Anaesth. 1995; 42(1): 12-5. (doi: 10.1007/BF03010564).

17. Harrison CA, Morris S, Harvey JS. Effect of ilioinguinal and iliohypogastric nerve block and wound infiltration with $0.5 \%$ bupivacaine on postoperative pain after hernia repair. $\mathrm{Br} J$ Anaesth. 1994; 72(6): 691-3. (doi: 10.1093/bja/72.6.691). 